\title{
Contribution of Broadcast Algorithms to Reactive Ad Hoc Routing
}

\author{
João Matos \\ Instituto Superior Técnico \\ INESC-ID \\ Email: jmatos@gsd.inesc-id.pt
}

\author{
Hugo Miranda \\ Universidade de Lisboa - Faculdade de Ciências \\ LaSIGE \\ Email: hmiranda@di.fc.ul.pt
}

\begin{abstract}
Most reactive routing protocols for ad hoc wireless networks rely on a broadcast algorithm known as flooding for route discovery. With flooding, many copies of each message are retransmitted unnecessarily resulting in a sub-optimal resource consumption. Given that mobile devices usually have limited power and computational resources, it is of the utmost importance to reduce the resource consumption of each route discovery. A number of alternatives to flooding have been presented but their impact on the performance of reactive routing protocols is not well understood. This paper compares and discusses the performance of a routing protocol for MANETs with three distinct broadcast algorithms.
\end{abstract}

Keywords-flooding; routing protocols; MANETs

\section{INTRODUCTION}

Mobile Ad Hoc Networks (MANETs) are wireless networks with no fixed infrastructure. In MANETs, nodes communicate using their wireless network interfaces, which have a limited transmission range unlikely to cover all the nodes in the network. Routing protocols are responsible for discovering a sequence of intermediate nodes (a route) that connects any two endpoints. Routing addresses non-trivial problems because it is assumed that $i$ ) nodes are not aware of the location of other nodes and $i$ i) nodes move, breaking the routes that have been found in the past. To save batteries, routes should present the lowest possible cost, usually evaluated by the number of nodes that compose them. A smaller route implies a lower number of retransmissions of each data packet, which has been shown to contribute for the increase of the devices' lifetime.

In reactive routing protocols, routes are discovered on-demand. When a node does not possess a route to the destination of some data packet, it triggers a route discovery procedure by broadcasting a route request message. This message must be propagated to the entire network, so that it reaches either the destination or some other node aware of a route for it. The most common broadcast algorithm is flooding (e.g. [1], [2]),

The work described in this paper was partially supported by Fundação para a Ciência e Tecnologia (FCT) through project PTDC/EIA-EIA/103751/2008 - PATI that is, to have all the nodes retransmitting the message when it is received for the first time. Given that all nodes retransmit the message, it is expected for the destination to receive multiple copies. In possession of the information accumulated by each of these copies, it is up to the destination to inspect, evaluate and select one or multiple routes and deliver them to the source.

Flooding may produce many concurrent retransmissions, what is known to cause unavailability problems and bandwidth loss [3]. Given that transmissions are one of the most power demanding operations performed by mobile devices, flooding also contributes to decrease the network lifetime. A number of alternative broadcast algorithms (e.g. [3]-[6]) have been proposed. Evaluation of these algorithms showed that it is possible to reduce the number of retransmissions in broadcast operations while maintaining a high delivery ratio.

The replacement of flooding by an alternative broadcast algorithm should bring to reactive routing protocols the same performance gains found elsewhere. However, broadcast algorithms achieve their fine performance by preventing some nodes from retransmitting. This impacts the amount and quality of the information made available to routing protocols, what in turn, may affect their route evaluation and selection criteria. In this paper, we study how three alternative broadcast algorithms impact the performance of a reactive routing protocol. The goal is to understand their impact on the quality of the routes selected and on the overall performance of the protocol. Lessons learned with these experiments can easily be applied in other protocols and applications, contributing for an increase in their performance, improve bandwidth usage and extend network lifetime.

\section{Broadcast Algorithms in Route Discovery}

Flooded messages are retransmitted once by each node, after being received for the first time. In an ideal environment, i.e. without collisions nor jitter and with a constant latency on each retransmission, the flooding of a route request permits to identify the optimal set of node disjoint routes. The set is optimal because each node will retransmit the first copy it received which is the one that traversed the smallest number of hops. The 
quality of each route can therefore be inferred from its arrival time at the destination.

Intuition suggests that the nice properties of flooding will in general hold even in a more realistic scenario with collisions and jitter. Although these factors are associated with degrading network conditions, overloaded nodes and congested network regions are likely to introduce additional delay in route request propagation thus reducing the probability of having new routes using them. Collisions, which in some cases can prevent message propagation, can also be seen as beneficial because they are more likely to occur in congested regions and therefore tend to divert new traffic. In summary, although affected by a number of factors, the earliest copies of a flooded route request received by any device are expected to be those that flowed more easily, with less congestion and hops and therefore are expected to describe the best routes.

However, flooding also presents some drawbacks. Transmissions' propagation time in the ether is very small. Therefore, nodes in proximity are likely to attempt to retransmit the message within a time frame that is too small for each to detect the others concurrent retransmission. As a result, a large number of collisions should be expected, specially in networks with a high node density. To avoid this issue each node postpones its retransmission by some random time. This delay can be harmful for the best routes' discovery as it can postpone good routes more than bad ones, obfuscating the additional delay suffered by routes traversing congested regions. In addition, flooding contributes to a degrading network performance both in terms of network lifetime and goodput, because of the large proportion of air time consumed by control messages.

The goal of broadcast algorithms is to save bandwidth and power by achieving delivery ratios comparable with those of flooding although with a smaller number of retransmissions. A key component for this effort is the algorithm run by each node that decides to retransmit or not the message. The following paragraphs briefly describe some retransmission criteria (see [7] for a survey):

Probabilistic criteria: Each node retransmits a message received for the first time with some probability $p$, where $p$ is a system-wide predefined constant. The probabilistic criteria tends not to adapt well to variations in node's density. In areas with fewer nodes, the prospect of all nodes deciding not to retransmit increases which may result on a premature end of the broadcast. Examples of algorithms using this criteria are [3], [4], [6], [8].

Counter-based criteria: The decision to retransmit is postponed after receiving the first copy of each message. During this period, nodes count the number of retransmissions they listen. Nodes decide to retransmit if the number of retransmissions listened is below some system-wide threshold. The definition of the waiting time varies. Examples are its random selection by each node (e.g. [3]) and a system-wide constant (e.g. [4], [8]).

Distance-based criteria: Considering an hop-by-hop propagation, the more distant the nodes are from the transmitter, more additional area their retransmissions will cover. Algorithms using this criteria try to select the more distant nodes. The distance between two nodes can be estimated using location information or the Received Signal Strength Indication (RSSI). Examples of applications of the distance-based criteria are to derive a decision to retransmit (e.g. [3]) or to set the expiration timer of a counter-based algorithm (e.g. [5]).

Neighbor knowledge criteria: Neighbor knowledge methods require each node to estimate the number of its 1 or 2-hop neighbors. This information is usually obtained by periodic exchanges of Heartbeat packets. This knowledge imposes a constant overhead in the network during periods where no retransmissions are required. Examples of this family of algorithms can be found in [9]. To address this problem, in some algorithms (e.g. [6]) nodes learn the network topology by piggybacking data messages. However, these algorithms tend not to cope well with node movement which may invalidate the topology information.

All of the above criteria focus on reducing the number of retransmissions required to broadcast a message. However, such a reduction can negatively affect the route selection process for two reasons: $i$ ) the number of routes to be discovered will be considerably smaller, accompanying the number of transmitters; and ii) broadcast algorithms select the transmitters that are more relevant according to their own criteria, which may conflict with the routing protocol expectations. In summary, the use of a broadcast algorithm other than flooding may reduce the probability of having the best routes discovered, as the nodes that compose them may not be selected for retransmission. On the other hand, they may not present congestion problems as severe as flooding, reducing route discovery delay and bandwidth consumption.

\section{Experimental SEtTings}

The approach to evaluate the impact of the broadcast algorithms in reactive routing protocols was to select a well known routing protocol and compare its performance when used with four broadcast algorithms. In addition to flooding (represented by the baseline routing protocol), two other well known broadcast algorithms were experimented combining respectively probabilistic with counter based criteria and counter with distance based criteria. The algorithms were selected from those that have exhibited better performance in previous evaluations (e.g. [5], [10]). A fourth broadcast algorithm 
was investigated and evaluated to address some limitations that were found in the remaining. The neighbor knowledge criteria was not considered in this study as it implies a periodic transmission from every node, an approach that is equally followed by proactive routing protocols and therefore, is out of the scope of this paper. This section describes the four variants of the routing protocol under evaluation.

$A O D V$ : The Ad hoc On-demand Distance Vector (AODV) [2] protocol was chosen as the baseline routing protocol. Considering the aspects that are relevant for our analysis, AODV's route discovery procedure is similar to many other reactive routing protocols, for example source routing ones like DSR [1]. In particular, it should be noted that route discovery messages are flooded to the network. The interested reader is referred to [2] for a complete description of AODV.

$A O D V+G$ : The version of the AODV protocol named $\mathrm{AODV}+\mathrm{G}$ replaces flooding by the $\operatorname{GOSSIP} 3(p, k, m)$ broadcast algorithm [4], which combines the probabilistic and counter-based criteria. In GOSSIP3, nodes retransmit with probability 1 if in the first $k$ hops from the source or with probability $p$ otherwise. If a node decides not to retransmit, it holds the message for a standard time interval. When that timer expires, the message will be retransmitted if less than $m$ copies of the message were listened. GOSSIP3 was previously experimented with AODV [4]. Results show that the replacement of flooding by GOSSIP3 reduced the number of route request retransmissions up to $35 \%$. As a consequence, AODV's performance improved significantly, specially in packet delivery ratio and delay although routes were 10\%-15\% longer.

$A O D V+P$ : This version of AODV replaces flooding by PAMPA [5], a distance and counter-based broadcast algorithm. In PAMPA, devices delay their retransmission for some time, determined by a function delay $(\mathrm{RSSI})=$ $k \times$ RSSI that multiplies the RSSI of the message by a constant. During the interval, the device counts the number of copies listened, and the retransmission will not occur if this number reaches a predefined threshold. Considering that the RSSI value decays with the increase of the distance between nodes, the ones located farther away obtain the lowest delay and are more likely to retransmit first. Therefore, it is predictable for AODV $+\mathrm{P}$ to obtain shorter routes, composed by nodes separated by very large distances.

$A O D V+P 2:$ Preliminary simulations with node movement showed that routes discovered using $\mathrm{AODV}+\mathrm{P}$ are rapidly invalidated. This was attributed to the smaller number of hops of its routes. If nodes are more distant from each other, they are more likely to move to some location outside the transmission range of the previous or next hop. To address this problem,

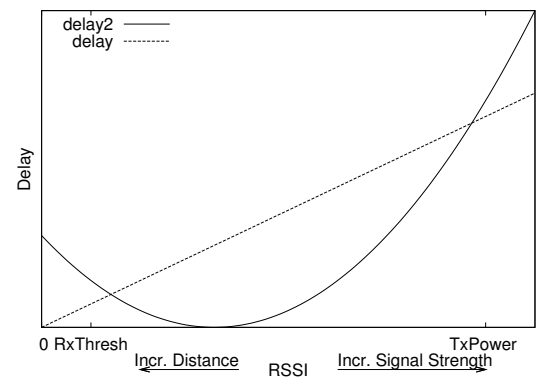

Figure 1. Delay functions of $\mathrm{AODV}+\mathrm{P}$ and $\mathrm{AODV}+\mathrm{P} 2$

$\mathrm{AODV}+\mathrm{P} 2$, a variant of $\mathrm{AODV}+\mathrm{P}$ was experimented. $\mathrm{AODV}+\mathrm{P} 2$ uses a quadratic delay $_{2}(\mathrm{RSSI})$ function that places its vertex closer to the centre of the device's transmission range. Therefore, AODV $+\mathrm{P} 2$ gives preference to nodes located at an intermediate distance within the sender's transmission range, thus less vulnerable to route invalidation.

A comparison between the functions delay and delay 2 is depicted in Fig. 1. In the figure, RxThresh is the lowest $R S S I$ that allows a node to listen a message and TxPower is the transmitted signal strength intensity.

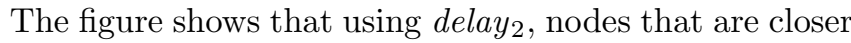
to some intermediate distance will set their timer to the minimum value, while in delay, the minimal waiting time is achieved at the limit of the transmission range.

$\mathrm{AODV}+\mathrm{P} 2$, like SSA [11] and ABR [12], attempts to infer link quality and route stability from RSSI. However, $i$ ) in contrast with $\mathrm{SSA}$ and $\mathrm{ABR}, \mathrm{AODV}+\mathrm{P} 2$ does not impose a burden on the network or on the device's batteries when no data traffic exists; $i i) \mathrm{AODV}+\mathrm{P} 2$ does not make any inferences of future node movement from past experiences and is therefore orthogonal to the movement model; and iii) SSA relies on a system wide RSSI threshold that will dictate the minimum link quality of all links in preferred routes. If no such route can be found, SSA will need to rerun the route discovery algorithm. To define an adequate delay $_{2}$ function, one must instead estimate the transmission signal strength and receiving threshold of the wireless network cards, which are part of the wireless interface specs. In $\mathrm{AODV}+\mathrm{P} 2$, the impact of an inaccurate estimation is limited to a slight bias of the nodes selected for retransmission. If a route exists, $\mathrm{AODV}+\mathrm{P} 2$ will find it at the first attempt.

\section{Evaluation}

The $n s-2$ network simulator was used to compare the impact of each broadcast algorithm on the traffic and on the routes selected by AODV. Simulations consider that nodes use an IEEE 802.11 network interface emulating Lucent's WaveLAN with a transmission range of $250 \mathrm{~m}$ at $2 \mathrm{Mb} / \mathrm{s}$. The two-ray ground reflection propagation 
model was used. This is a deterministic propagation model, where all nodes within transmission range of the sender receive the message. This concentrates the factors that influence the performance of the routing protocol on the Link Layer and Routing levels, which are those that can be distinctly affected by the broadcast algorithms and therefore, facilitate the analysis of our experiments. The impact of the non-determinism of the wireless media is outside the scope of this paper (see [13], [14]).

Distinct node densities and average route lengths are evaluated by testing a constant number of 200 nodes on networks with $1500 m \times 1000 m$ (High Density-HD), $2500 \mathrm{~m} \times 1000 \mathrm{~m}$ (Medium Density-MD) and $3500 \mathrm{~m} \times$ $1000 m$ (Low Density-LD). In all tests, 30 connections between randomly chosen nodes are initiated at a random time. The connections use the UDP protocol to send 512 bytes packets in one direction. The sensitivity of the algorithms to traffic is evaluated by experimenting both $2 \mathrm{pkts} / \mathrm{s} /$ connection (in HT-High Traffic) or $0.2 \mathrm{pkts} / \mathrm{s} /$ connection (in LT-Low Traffic).

Nodes move according to the random waypoint model, at speeds between $1 \mathrm{~m} / \mathrm{s}$ and $2 \mathrm{~m} / \mathrm{s}$ and between $5 \mathrm{~m} / \mathrm{s}$ and $12 \mathrm{~m} / \mathrm{s}$. The pause time was set to $120 \mathrm{~s}$ in all tests. The sensitivity of the algorithms to movement is compared with a scenario where nodes are uniformly deployed and do not move. Each simulation is run for $2800 \mathrm{~s}$, of which the first $1000 \mathrm{~s}$ are a warm-up period used to shuffle nodes. Results depict the average of 40 simulations for each combination of broadcast algorithm, topology class, traffic intensity and speed.

With exception of flooding, all broadcast algorithms experimented require the configuration of some parameters. Following author suggestions, a $\operatorname{GOSSIP3}(0.65,1,1)$ configuration was used [4]. The delay and delay 2 functions used in AODV $+\mathrm{P}$ and AODV $+\mathrm{P} 2$ depend on the scale of the RSSI, which, in $n s-2$ is in Watts. Simulations use functions $\operatorname{delay}(x)=5 \times 10^{6} \times x$ and $\operatorname{delay}_{2}(x)=\frac{1}{25}\left(4\left(10^{8} \times x\right)^{2}-28 \times 10^{8} \times x+49\right)$ experimentally devised.

Metrics: The four algorithms are compared using the following metrics. The route discoveries metric counts the number of these operations initiated. It evaluates the quality of the routes found, given that a new route discovery must be initiated whenever the existing route is invalidated or was not found in a previous attempt. Results for this metric are presented in proportion of the values obtained for AODV.

The retransmissions metric counts the accumulated number of transmissions for all nodes at the link layer level. It gives a good indication of the total traffic (data and route maintenance) produced by the protocols.

The route length metric averages the number of hops of all the routes used. This metric is affected by the network region size but also by the ability of each algorithm to select the most distant nodes for retransmission.

The overall efficiency of the routing protocols is evaluated by the delivery ratio metric, defined by the proportion of transmitted data packets that are successfully delivered to the destination. Broadcast algorithms have a direct impact on the delivery ratio as they: $i$ ) compete with data packets for air time, and $i$ i) influence the route selection process and therefore, route stability.

Simulation Results: The delivery ratio results are depicted in Fig. 2. Results show that all protocols achieve a delivery ratio close to 1 with low traffic and without node movement but degrades with the increase of traffic and average speed. This is justified by the larger contention suffered by data packets when traffic increases. Additionally node movement may lead to more route discovery operations which induce additional traffic.

The advantages of replacing flooding are visible by comparing Fig. 2(a) and 3(b). These figures clearly show that when nodes do not move it is possible to obtain comparable or higher delivery ratios using $60 \%$ or less retransmissions. The most remarkable difference on the delivery ratio happens in HD-HT, which are the most stressful network conditions, as nodes are competing for air time in a smaller region. In these scenarios, the reduction of the contention achieved by $\mathrm{AODV}+\mathrm{P}$ from its small number of retransmissions per route discovery, allows it to obtain a higher delivery ratio.

The differences between the node selection criteria used by the algorithms is visible on Fig. 3(a). The figure shows that the gains on the number of retransmissions of $\mathrm{AODV}+\mathrm{P}$ and $\mathrm{AODV}+\mathrm{P} 2$ are not reflected in route stability, as the number of route discoveries exceeds those of AODV and AODV+G. The peak that these protocols present in the number of route discoveries for Low Density-Low Traffic scenarios is attributed to: $i$ ) a failure of the broadcast algorithms in delivering the route requests to some destinations, which results in periodic repetitions of the route discovery; and $i i$ ) the concentration of traffic on some nodes who thus become unable to forward all packets, which is interpreted by AODV as a route failure.

$\mathrm{AODV}+\mathrm{P}$ and AODV $+\mathrm{P} 2$ are the protocols that respectively tend to present the lowest and the highest average route length. This comes from the node selection criteria followed by these protocols, which tend to select respectively the nodes that are more distant from the transmitter (thus resulting in the shortest routes) or at some intermediate position. Node selection in AODV and $\mathrm{AODV}+\mathrm{G}$ follows the random criteria and therefore tend to find routes with an average length. Figure 3(c) confirms previous results with $\mathrm{AODV}+\mathrm{G}$ that showed a small increase in route length over AODV [4] and that can be attributed to the smaller number of routes 


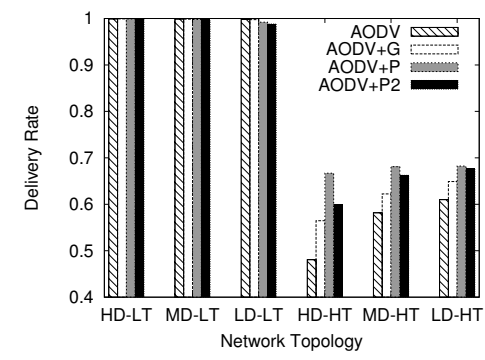

(a) No movement

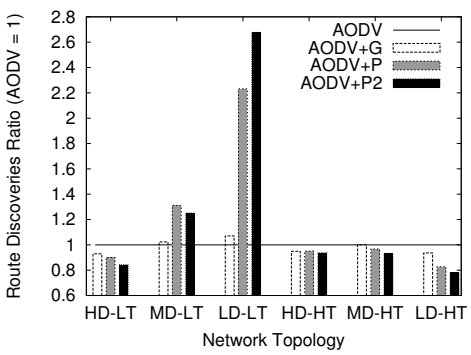

(a) Route discoveries

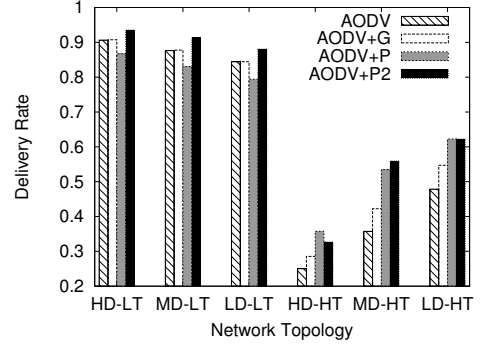

(b) $1-2 m / s$

Figure 2. Delivery Ratio

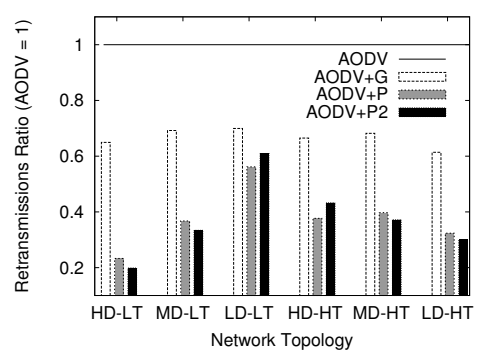

(b) Retransmissions

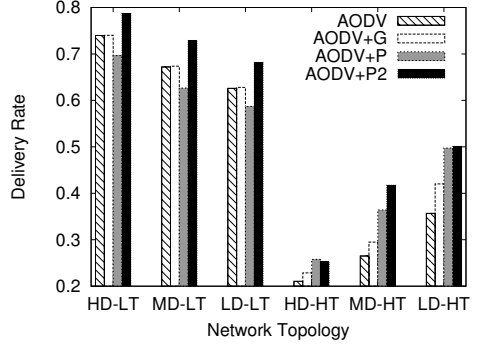

(c) $5-10 \mathrm{~m} / \mathrm{s}$

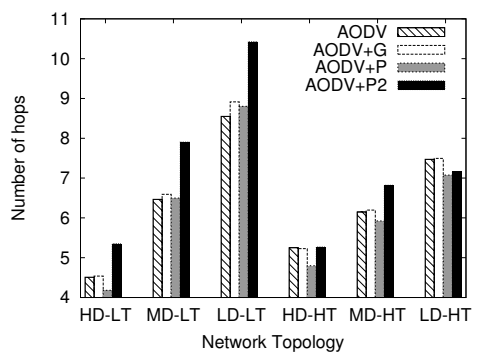

(c) Route length

Figure 3. Simulation results without node movement

discovered by AODV $+\mathrm{G}$, increasing the probability of not discovering one with the minimal length.

Figures 4 and 5 depict the simulation results in movement scenarios. The similar patterns exhibited by the figures for each metric suggest that the relative performance of the protocols does not change with node speed and that they can be evaluated together. The differences in the scales are attributed to the additional difficulties posed by the node movement at higher speeds, which results in more frequent changes in the topology and therefore, in an increased number of broken routes.

In general, $\mathrm{AODV}+\mathrm{P} 2$ exhibits the best performance in the delivery ratio and retransmissions. These results are attributed to the small number of route discoveries, which are the biggest contributor for the number of retransmissions. In contrast, $\mathrm{AODV}+\mathrm{P} 2$ routes are longer than those of the remaining. This leads to the conclusion that the selection of nodes at intermediate distance effectively contributes for an increased route stability. In the scenarios where AODV $+\mathrm{P} 2$ 's average route length is comparable with other protocols, its smaller number of retransmissions contributes to reduce contention.

As expected, node selection criteria of $\mathrm{AODV}+\mathrm{P}$ is not adequate for scenarios with node movement. Figures 4(c) and $5(\mathrm{c})$ confirm that AODV $+\mathrm{P}$ always selects the routes with the lowest number of hops. However, this is counterproductive when nodes move frequently and therefore can easily move outside the transmission range of the previous hop. In high traffic scenarios, contention and collision increase and it is important to keep the amount of control messages to a minimum. In this case, AODV $+\mathrm{P}$ still presents a good performance.

With exception of the number of retransmissions, $\mathrm{AODV}$ and $\mathrm{AODV}+\mathrm{G}$ tend to present comparable performances. This suggests that the random node selection criteria followed by $\mathrm{AODV}+\mathrm{G}$ produces roughly equivalent results to the random jitter introduced by flooding. The benefits of AODV $+\mathrm{G}$ on the delivery ratio are more visible in high traffic, where the savings of broadcast algorithms increase the delivery ratio.

\section{Conclusions}

In MANETs, routes must be discovered by having intermediate hops to forward route request messages. In original routing protocols, the forwarding of the route request was performed by all nodes in the network, a process known as flooding. This paper reports the experiments with three alternative broadcast algorithms, that considerably reduce the number of forwarders although following different algorithms for node selection. Simulation results suggest that in general, any of this algorithms outperforms flooding in delivery ratio and number of retransmissions. Finally, the paper highlighted the performance differences of the algorithms when experimented in different network conditions. 


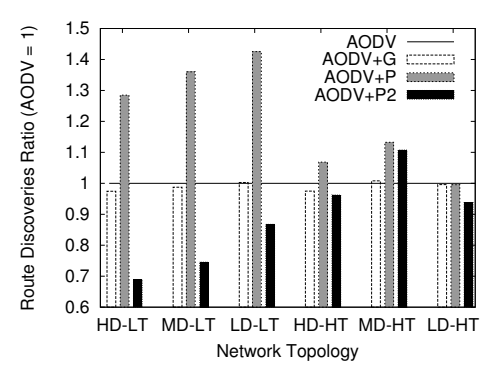

(a) Route discoveries

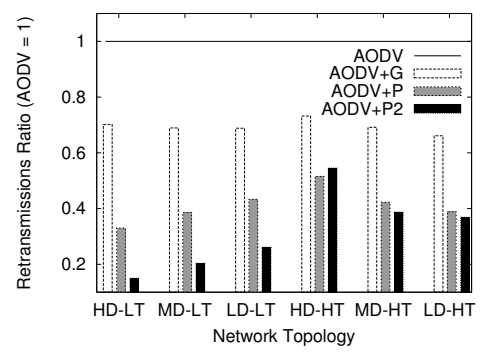

(b) Retransmissions

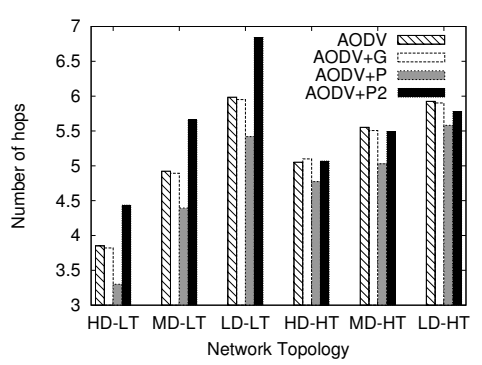

(c) Route length

Figure 4. Simulations results for speeds $1-2 m / s$

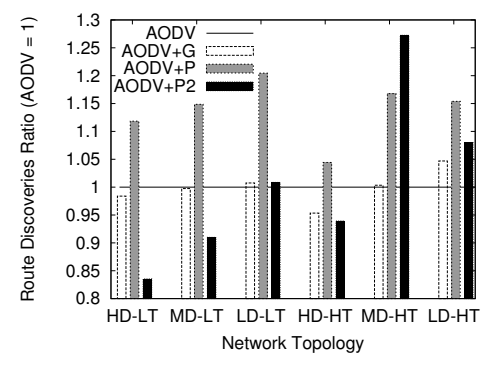

(a) Route discoveries

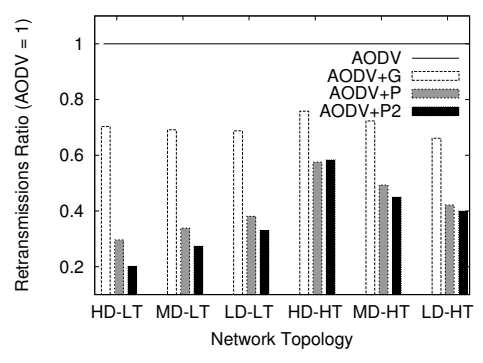

(b) Retransmissions

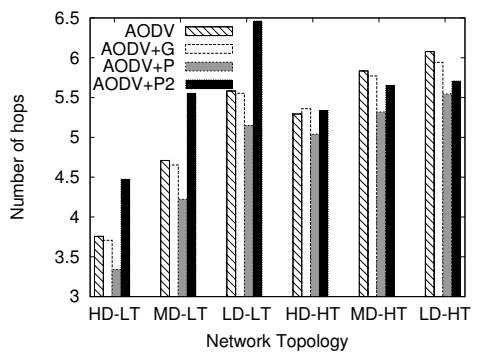

(c) Route length

Figure 5. Simulations results for speeds $5-12 \mathrm{~m} / \mathrm{s}$

\section{REFERENCES}

[1] D. B. Johnson, D. A. Maltz, and J. Broch, Ad Hoc Networking. Addison-Wesley, 2001, ch. DSR: The Dynamic Source Routing Protocol for Multi-Hop Wireless Ad Hoc Networks, pp. 139-172.

[2] C. E. Perkins and E. M. Royer, "Ad-hoc on-demand distance vector routing," in 2nd Works. on Mobile Comp. Systems and Applications, 1999, pp. 90-100.

[3] Y.-C. Tseng, S.-Y. Ni, Y.-S. Chen, and J.-P. Sheu, "The broadcast storm problem in a mobile ad hoc network," Wireless Networks, vol. 8, no. 2/3, pp. 153-167, 2002.

[4] Z. J. Haas, J. Y. Halpern, and L. Li, "Gossip-based ad hoc routing," in Procs. of the 21st Joint Conf. of the IEEE Comp. and Comm. Societies (INFOCOM 2002), vol. 3, 2002, pp. 1707-1716.

[5] H. Miranda, S. Leggio, L. Rodrigues, and K. Raatikainen, "A power-aware broadcasting algorithm," in 17th IEEE Int'l Symp. on Personal, Indoor and Mobile Radio Comm. (PIMRC'06), 2006.

[6] P. Kyasanur, R. R. Choudhury, and I. Gupta, "Smart gossip: An adaptive gossip-based broadcasting service for sensor networks," in Int'l Conf. on Mobile Adhoc and Sensor Systems (MASS), 2006, pp. 91-100.

[7] B. Garbinato, A. Holzer, and F. Vessaz, "Context-aware broadcasting approaches in mobile ad hoc networks," Computer Networks, vol. 54, no. 7, 2010.
[8] V. Drabkin, R. Friedman, G. Kliot, and M. Segal, "RAPID: Reliable probabilistic dissemination in wireless ad-hoc networks," Technion - Israel Institute of Technology, Tech. Rep. CS-2006-19, 2006.

[9] W. Peng and X.-C. Lu, "On the reduction of broadcast redundancy in mobile ad hoc networks," in Proc, of the 1st ACM Int'l Symp. on Mobile ad hoc networking 8 computing (MobiHoc '00), 2000, pp. 129-130.

[10] B. Garbinato, A. Holzer, and F. Vessaz, "Six-shot broadcast: A context-aware algorithm for efficient message diffusion in MANETs," in Procs. of the On the Move to Meaningful Internet Systems Conference (OTM 2008), ser. LNCS, vol. 5331. Springer, 2008, pp. 625-638.

[11] R. Dube, C. D. Rais, K.-Y. Wang, and S. K. Tripathi, "Signal stability-based adaptive routing (ssa) for ad hoc mobile networks," Personal Communications, vol. 4, no. 1, pp. 36-45, 1997.

[12] C.-K. Toh, "Associativity-based routing for ad hoc mobile networks," Wireless Personal Comm., vol. 4, pp. 103-139, 1997.

[13] T. Yang, M. Ikeda, G. De Marco, and L. Barolli, "Performance behavior of AODV, DSR and DSDV protocols for different radio models in ad-hoc sensor networks," in Int'l Conf. on Parallel Processing (ICPPW 2007) Works., 2007.

[14] J. Matos and H. Miranda, "Ad hoc routing under randomised propagation models," in Actas do INFORUM Simpósio de Informática, 2010, pp. 379-390. 